\title{
Online resources for platelet-rich plasma injections for orthopedic therapy: What are patients reading? A survey of Turkish websites
}

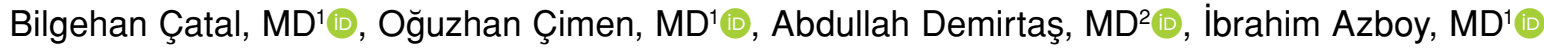 \\ ${ }^{1}$ Department of Orthopedics and Traumatology, Medipol University Faculty of Medicine, Istanbul, Turkey \\ ${ }^{2}$ Department of Orthopedics and Traumatology, Medeniyet University Faculty of Medicine, Istanbul, Turkey
}

Nowadays, thanks to the internet service, accessing information is simple and fast. Global internet access rate was $25 \%$ in 2009 whereas this rate was reported as $58.8 \%$ in 2019. ${ }^{[1]}$ The situation is similar in Turkey. Turkish Statistical Institute reported an internet usage rate of $72.9 \%$ in people aged $16-74$ years in $2018 .{ }^{[2]}$ The internet has become a primary information tool to investigate diseases and treatment choices since it became readily accessible even from mobile devices today. A study from the US reported that more than half of adult Americans perform researches about their health-related questions via internet. ${ }^{[3]}$ The main reasons for using the internet are to evaluate alternative treatment methods, confirm the information provided by doctors, and answer the questions about their conditions. ${ }^{[4]}$

Received: November 20, 2019

Accepted: March 08, 2020

Published online: September 11, 2020

Correspondence: Bilgehan Çatal, MD. Medipol Üniversitesi Tıp Fakültesi Ortopedi ve Travmatoloji Anabilim Dalı, 34810 Beykoz, Istanbul, Türkiye.

E-mail: drbilgehancatal@yahoo.com

Doi: $10.5606 /$ ehc. 2020.72391

Citation: Çatal B, Çimen O, Demirtaş A, Azboy I. Online resources for platelet-rich plasma injections for orthopedic therapy: What are patients reading? A survey of Turkish websites. Jt Dis Relat Surg 2020;31(3):582-588

(02020 All right reserved by the Turkish Joint Diseases Foundation

This is an open access article under the terms of the Creative Commons Attribution-NonCommercial License, which permits use, distribution and reproduction in any medium, provided the original work is properly cited and is not used for commercial purposes (http://creativecommons.org/licenses/by-nc/4.0/).

\section{ABSTRACT}

Objectives: This study aims to evaluate the quality, accuracy, and readability of Turkish online resources for platelet-rich plasma (PRP) injections for orthopedic therapy.

Patients and methods: In this retrospective study, online searches using Google, Yandex, and Yahoo search engines were performed on 21-22-23 May 2019, respectively. "Platelet-rich plasma", "platelet-rich plasma treatment", "PRP", and "PRP treatment" were entered in Turkish into these three search engines. The first 50 websites from each search were collected. The quality and accuracy of online information related to PRP injections for orthopedic therapy were evaluated by three reviewers with the use of scoring criteria specific to PRP. The Flesch-Kincaid (FK) score was used to determine readability.

Results: Eighty-six unique websites were evaluated. The average quality and accuracy scores of all websites were $7.1 \pm 4.3$ out of a maximum of 25 points and $7.3 \pm 2$ out of a maximum of 12 points, respectively. The average FK score of all websites was 10.8 \pm 2.2 . Only 27 websites $(31.4 \%$ ) had a FK score that was at or below the eighth-grade level. There were no significant differences among the mean scores of websites categorized by search terms, search results ranking, owners or reading level for both quality and accuracy scores.

Conclusion: The information regarding PRP usage in orthopedic conditions provided by Turkish online resources has low quality and low accuracy ratings and is also difficult to read.

Keywords: Accuracy, health information, internet, online search, platelet-rich plasma, quality, readability.

Health-related information from internet sources has not been regulated adequately and unfortunately may contain misleading information. Basic concerns for healthcare information obtained from the internet are the possibility of medically incorrect information, misleading information due to commercial interest, and that texts can be above the patient's reading level. Previous studies reported that information about orthopedic conditions provided by online resources 
may have low-quality and be inaccurate with difficult texts for the patient to read. ${ }^{[5-8]}$ When this misleading information obtained from internet sources and the medical information given by the doctor contradict, the trust bond between the patient and the doctor may be disturbed..$^{[9]}$ Also, correcting the inaccurate information obtained from online resources by the patients became a routine chore for the doctors so that the treatment progress is not affected.

Biological treatments used in orthopedic conditions have gained popularity in recent years and created a large commercial market for those therapies. ${ }^{[10]}$ Amongst biological treatment options, platelet-rich plasma (PRP) injections became one of the most popular due to their ease of preparation that can be performed in an office setting and their relative cost-efficiency. ${ }^{[11]}$ A global industry analysis study about PRP usage showed that the annual market share in PRP treatments used in orthopedics was about 160 million dollars in 2015 and is expected to increase by $11 \%$ each year, reaching an annual market share of 451 million dollars by $2024 .{ }^{[12]}$ It may be assumed that the huge amount of information on internet may affect this enormous market share.

Our first hypothesis is that the quality and accuracy of the information from online sources are affected by keywords, search engine order, and type of website. Our second hypothesis is that increased accuracy and quality of online information decrease readability. Therefore, in this study, we aimed to evaluate the quality, accuracy, and readability of Turkish online resources for PRP injections for orthopedic therapy.

\section{PATIENTS AND METHODS}

In this retrospective study conducted at Koşuyolu Medipol Hospital, online searches using Google, Yandex, and Yahoo search engines were performed on 21-22-23 May 2019, respectively. Google, Yandex, and Yahoo search engines are the first three search engines used in Turkey with the usage rates of $87.7 \%, 9.85 \%$, and $1.32 \%$, respectively. ${ }^{[13]}$ We used "plateletten zengin plazma", which is platelet-rich plasma in Turkish, "plateletten zengin plazma tedavisi", which is platelet-rich plasma treatment in Turkish, "PRP", and "PRP tedavisi", which is PRP treatment in Turkish as keywords. The settings in each search engine were set to show only Turkish websites and all searches were performed in Istanbul, Turkey. Cookies were cleared before each search. This step is important as the online patient resources on the search results of search engines might change due to the user's previous searches and advertising choices that are stored in cookies. A previous study reported that people who perform online research about health-related issues usually scan the first few pages of search engine results. ${ }^{[14]}$ Therefore, we included the first 50 website results shown on the search engine. Duplicates (same pages with different keywords or different search engine results), medical articles for healthcare professionals, sites that deal with PRP therapy other than orthopedic conditions, broken links, video links, and paid websites were excluded. Websites including login requirement were excluded because although $26 \%$ of online users looking for health information reported being asked to pay for access, only $2 \%$ actually made such a payment. ${ }^{[15]}$ The study was conducted in accordance with the principles of the Declaration of Helsinki.

The websites included in the study were categorized according to their sources as websites by healthcare providers (such as hospitals, clinics, and personal websites of medical professionals) and sites by non-healthcare providers (such as news websites, online health sites, blogs, and commercial biomedical company websites). Websites were ranked according to their order in the search results in all three search engines and classified as results between 1-10, 11-20, 21-30, 31-40, and 41-50.

The quality and accuracy of each website were assessed using a previously published scoring technique. ${ }^{[5,6,16]}$ The website addresses were randomly compiled into an Excel file. The websites were assessed by three board-certified orthopedic surgeons with at least five years of experience and who were blinded to the keywords and search engines in terms of quality and accuracy of the provided material. A current review of PRP usage in orthopedics ${ }^{[17]}$ was read by the researchers before assessment. The quality score was determined using an assessment score from a similar previous study ${ }^{[18]}$ The 25-point scoring criteria were based on the guidelines of the American Academy of Orthopedic Surgeons ${ }^{[18]}$ which included the definition, composition, rationale for usage, applications, and a general understanding of PRP (Table I). For each element discussed in the website content, the website was given 1 point while 0 point was given if the website did not include any of the mentioned elements. The mean quality score for each website with a maximum of 25 was calculated by averaging the independent scores from the three evaluators. Reviewers scored content accuracy from 1 to 4 , with 1 showing <25\% accuracy, 2 for $25-50 \%$ accuracy, 3 for $51-75 \%$ accuracy, and 4 for $76-100 \%$ accuracy. Accuracy scores calculated for each website 


\section{RESULTS}

The initial search yielded 600 websites. After removing duplicates $(n=267)$ and websites that met the exclusion criteria $(n=247)$, the final group consisted of 86 websites that discussed PRP injections for orthopedic therapy. The flowchart diagram is given in Figure 1.

Of the 86 unique websites, 13 were categorized under "plateletten zengin plazma" (platelet-rich plasma), 26 were categorized under "plateletten zengin plazma tedavisi" (platelet-rich plasma treatment), 18 were categorized under "PRP", and 29 were categorized under "PRP tedavisi" (PRP treatment). Out of 86 websites included in the study, $53(61.6 \%)$ were from healthcare resources (hospitals, clinics or personal websites of medical professionals) whereas 33 (38.4\%) were from non-healthcare resources (newspapers, magazines, blogs, online healthcare websites, or biotechnology companies). When sorted by the highest search order, 14 websites appeared between one and 10, 22 websites appeared between 11 and 20, 12 websites appeared between 21 and 30, 19 websites appeared between 31 and 40, and 19 websites appeared between 41 and 50 .
The average quality score of all websites was $7.1 \pm 4.3$ out of a maximum of 25 points (range, 1-24). The quality rating was reliable among the reviewers with an intraclass correlation coefficient of 0.82 . There were no significant differences among the mean quality scores of websites categorized by search terms $(p=0.06)$, search results ranking $(p=0.18)$ owners $(p=0.16)$ or reading level $(p=0.65)$. The summary of quality results was given in Table II.

The average accuracy score of all websites was $7.3 \pm 2$ out of a maximum of 12 points (range, $3-12$ ). The accuracy rating was reliable among the reviewers with an intraclass correlation coefficient of 0.74 . Only two websites $(2.3 \%)$ received a maximum score of 12. There were no significant differences among the mean quality scores of websites categorized by search term $(\mathrm{p}=0.47)$, search results ranking $(\mathrm{p}=0.56)$, owners $(p=0.46)$ or reading level $(p=0.54)$. The summary of the accuracy results was given in Table III.

The average FK grade score of all websites was 10.8 \pm 2.2 (range, 5.6-18.8). Only 27 websites (31.4\%) had a FK grade score that was at or below the eighthgrade level.

\begin{tabular}{|c|c|c|c|c|}
\hline \multicolumn{5}{|c|}{$\begin{array}{l}\text { TABLE II } \\
\text { Summary of quality results }\end{array}$} \\
\hline & $\mathrm{n}$ & Mean $\pm S D$ & Range & $p$ \\
\hline All websites & 86 & $7.1 \pm 4.3$ & $1-24$ & \\
\hline Search term & & & & 0.06 \\
\hline "Plateletten zengin plazma" & 13 & $9.3 \pm 6.7$ & $2-24$ & \\
\hline "Plateletten zengin plazma tedavisi" & 26 & $7.8 \pm 3.6$ & $3-18$ & \\
\hline Platelet-rich plasma & 18 & $5.5 \pm 3.6$ & $1-14$ & \\
\hline Platelet-rich plasma treatment & 29 & $6.3 \pm 3.4$ & $2-20$ & \\
\hline \multicolumn{5}{|l|}{ Highest search result ranking } \\
\hline $1-10$ & 14 & $9.4 \pm 6.2$ & $2-24$ & 0.18 \\
\hline $11-20$ & 22 & $7 \pm 3.2$ & $2-17$ & \\
\hline $21-30$ & 12 & $7.7 \pm 5.4$ & $1-20$ & \\
\hline $31-40$ & 19 & $5.9 \pm 2.5$ & $2-11$ & \\
\hline $41-50$ & 19 & $6.3 \pm 4$ & $2-18$ & \\
\hline Owners of the websites & & & & 0.16 \\
\hline Healthcare provider & 53 & $7.3 \pm 3.8$ & $2-24$ & \\
\hline Non-healthcare provider & 33 & $6.8 \pm 5.1$ & $1-20$ & \\
\hline Reading level & & & & 0.65 \\
\hline Written at or below an eighth-grade reading level & 27 & $7.2 \pm 4$ & $2-20$ & \\
\hline Written above an eighth-grade reading level & 59 & $7 \pm 4.4$ & $1-24$ & \\
\hline
\end{tabular}




\begin{tabular}{|c|c|c|c|c|}
\hline $\begin{array}{c}\text { TABLE III } \\
\text { Summary of accurac }\end{array}$ & resu & & & \\
\hline & $\mathrm{n}$ & Mean \pm SD & Range & $p$ \\
\hline All websites & 86 & $7.3 \pm 2$ & $3-12$ & \\
\hline Search term & & & & 0.47 \\
\hline "Plateletten zengin plazma" & 13 & $7.6 \pm 2.9$ & $4-12$ & \\
\hline "Plateletten zengin plazma tedavisi" & 26 & $7.7 \pm 1.7$ & $4-11$ & \\
\hline Platelet-rich plasma & 18 & $6.8 \pm 2.1$ & 3-11 & \\
\hline Platelet-rich plasma treatment & 29 & $7.1 \pm 1.5$ & $4-10$ & \\
\hline Highest search result ranking & & & & 0.56 \\
\hline $1-10$ & 14 & $8.1 \pm 2.1$ & $5-12$ & \\
\hline $11-20$ & 22 & $7.3 \pm 2$ & $4-11$ & \\
\hline $21-30$ & 12 & $7.1 \pm 2.6$ & 3-11 & \\
\hline $31-40$ & 19 & $7.3 \pm 1.8$ & $4-10$ & \\
\hline $41-50$ & 19 & $7.3 \pm 1.9$ & $5-11$ & \\
\hline Owners of the websites & & & & 0.46 \\
\hline Healthcare provider & 53 & $7.4 \pm 1.8$ & $4-12$ & \\
\hline Non-healthcare provider & 33 & $7.1 \pm 2.2$ & $3-12$ & \\
\hline Reading level & & & & 0.54 \\
\hline Written at or below an eighth-grade reading level & 27 & $7.5 \pm 2.2$ & $4-11$ & \\
\hline Written above an eighth-grade reading level & 59 & $7.2 \pm 1.9$ & $4-12$ & \\
\hline
\end{tabular}

\section{DISCUSSION}

To the best of our knowledge, this is the first study that evaluated Turkish online resources for PRP treatment in orthopedic conditions. The most important finding of this study is that the information regarding PRP usage in orthopedic conditions provided by Turkish online resources has low quality and accuracy ratings and is also difficult to read.

Patients are more likely to refer to online resources for health-related information day by day. ${ }^{[25]}$ This increase causes concerns about the accuracy and reliability of the information provided by online resources. Although the internet has become a primary source for orthopedics-related information for patients, previous studies also reported that the information provided by online orthopedic website resources may have low quality and be inaccurate with difficult texts for the patient to read. ${ }^{[5-8]}$

Our study showed that the information from Turkish online resources about using PRP in orthopedic treatments also has low quality. The mean quality score for Turkish online resources was found to be $7.1 \pm 4.3$ out of 25 points. Ghodasra et al. ${ }^{[16]}$ assessed US-based online resources using the same quality score for the usage of PRP in orthopedic treatments. They reported a mean quality score of $9.4 \pm 3.4$ for studies on other orthopedic subjects using a similar methodology, including mean quality scores of $9.5 \pm 5.1$ in shoulder instability, $7.4 \pm 4.4$ in cartilage defects, $10.6 \pm 40$ in hallux valgus, $12 \pm 7.2$ in distal radius fractures, $11.9 \pm 6.1$ in lateral epicondylitis, and $11.4 \pm 5.5$ in trigger finger..$^{[5-8,26,27]}$ All the studies mentioned above reported a significantly better quality score in healthcare provider websites compared to other non-healthcare related websites. However, the present study did not show any such difference in Turkish websites. The reason that the Turkish online resources about PRP in orthopedic treatments have lower quality scores compared to other studies could be that the healthcare provider websites do not have high-quality information. As opposed to our initial hypothesis, the quality score was not affected by any of the variables we assessed.

The mean accuracy score for Turkish online resources was calculated as $7.3 \pm 2$ out of 12 points. A US-based similar study reported an average accuracy score of $7.9 \pm 2.9$ points. ${ }^{[16]}$ Again, as opposed to our initial hypothesis, the accuracy score was also not affected by any of the assessed variables. Our study shows that the information found in Turkish online 
resources about PRP in orthopedic treatments has a relatively higher accuracy rating compared to quality.

Other studies that use a similar methodology for the assessment of online resources about different orthopedic subjects also reported relatively higher accuracy ratings. The mean accuracy score was calculated as $10.2 \pm 1.4$ points in hallux valgus, $11.7 \pm 0.6$ in cartilage defects, and $8.61 \pm 2.6$ in shoulder instability out of $12 .{ }^{[5-7]}$ Our study and the previous studies showed better accuracy scores compared to quality. This is possibly due to the limited information provided. For example, a website accurately describing usage of PRP only in knee conditions and not in any other orthopedic pathologies might cause a higher accuracy score with a lower quality score. Inadequate information, even with full accuracy, can be misleading for the patients. For this reason, we think that PRP information provided by online resources should be on a wider scale.

The majority of the websites we reviewed (59\%) had a readability level above the eighth grade, which is above the recommended readability level for patients. A similar situation was also present in previous studies. This rate was reported as $87 \%$ in shoulder instability, 98\% in cartilage defects, and $79 \%$ in hallux valgus. ${ }^{[5-7]}$ Online medical information about orthopedic subjects had a lower readability rating. We found that the accuracy and quality scores were not affected by FK grade levels. This would mean that it is possible to write high-quality and accurate information using a more understandable language. We believe that online medical resources should be written in a simpler language for patients to understand what they read.

Our study demonstrates that improvement is needed in the Turkish online sources about PRP treatment for orthopedic conditions. We believe that two main issues need to be addressed to solve this situation. Unlike previous studies, we did not find any difference between healthcare providers' and non-healthcare providers' websites according to quality and accuracy scores. Firstly, healthcare providers should rearrange the information about the use of PRP in orthopedic treatment on their websites. The current literature and guidelines should be referenced, and a simple language should be used. In addition, when considering rapid development in biological treatments, the websites should be updated periodically, and the last update dates should be specified. Secondly, we observed that most of the information about PRP usage in orthopedics from the Turkish online resources offers only a limited perspective. We think that online healthcare information should be given in all aspects. Because of that, objective information about PRP treatment such as it is still investigational, has possible side effects and is a costly treatment should be included in online resources. ${ }^{[28,29]}$ We believe that by making these arrangements, Turkish websites can provide better quality, more accurate, and objective information to the patients.

This study has several limitations. The first limitation is that only four search terms were used even though patients might use different terms when searching on websites. Also, only three search engines were used. Using different keywords and search engines may alter the results. Moreover, the study was performed in a relatively short period. Because the internet is a very dynamic environment, we were able to assess only a single snapshot of the websites in question. The study period might affect the study results. Furthermore, the location where the web search is performed can change the search results and order. Different results may appear in web searches using the same search engine and the same keyword from a different city. However, in the current study, we evaluated the first 50 results for each search engine and each keyword; therefore, we think that this difference is negligible. In the current study, we used the FK formula, which is the most commonly used method in evaluating readability. Turkish adapted equivalents of this method were also described and readability values may differ when these methods are used. ${ }^{[30]}$ Finally, only Turkish websites were included in our study. We believe that the majority of our patients only read Turkish online resources yet there is a small number of patients who can read English resources as well.

In conclusion, to our knowledge, this is the first study to assess the quality, accuracy, and readability of information on Turkish websites regarding PRP injections for orthopedic therapy. The information regarding PRP usage in orthopedic conditions provided by Turkish online resources has low quality and low accuracy ratings and is also difficult to read. The quality and accuracy of the information from Turkish online sources are not affected by keywords, search engine order, type of website, or reading level. The findings of this study may influence the website owners regarding the data presented in their websites. Furthermore, physicians should inform patients that many online patient resources have poor quality and accuracy and can be difficult to read. We believe that the data presented in this study may allow better communication between physicians and patients. 


\section{Declaration of conflicting interests}

The authors declared no conflicts of interest with respect to the authorship and/or publication of this article.

\section{Funding}

The authors received no financial support for the research and/or authorship of this article.

\section{REFERENCES}

1. Internet Usage Statistics. Available at: https://www. internetworldstats.com/stats.htm [Accessed: June 24, 2019].

2. Hanehalkı Bilişim Teknolojileri (BT) Kullanım Araştırması, 2018. Available at: http://www.tuik.gov.tr/ PreHaberBultenleri.do?id=27819 [Accessed: June 24, 2019].

3. Amante DJ, Hogan TP, Pagoto SL, English TM, Lapane KL. Access to care and use of the Internet to search for health information: results from the US National Health Interview Survey. J Med Internet Res 2015;17:e106.

4. Castleton K, Fong T, Wang-Gillam A, Waqar MA, Jeffe DB, Kehlenbrink L, et al. A survey of Internet utilization among patients with cancer. Support Care Cancer 2011;19:1183-90.

5. Garcia GH, Taylor SA, Dy CJ, Christ A, Patel RM, Dines JS. Online resources for shoulder instability: what are patients reading? J Bone Joint Surg [Am] 2014;96:e177.

6. Wang D, Jayakar RG, Leong NL, Leathers MP, Williams RJ, Jones KJ. Evaluation of the quality, accuracy, and readability of online patient resources for the management of articular cartilage defects. Cartilage 2017;8:112-8.

7. Tartaglione JP, Rosenbaum AJ, Abousayed M, Hushmendy SF, DiPreta JA. Evaluating the quality, accuracy, and readability of online resources pertaining to hallux valgus. Foot Ankle Spec 2016;9:17-23.

8. Dy CJ, Taylor SA, Patel RM, Kitay A, Roberts TR, Daluiski A. The effect of search term on the quality and accuracy of online information regarding distal radius fractures. J Hand Surg Am 2012;37:1881-7.

9. Silberg WM, Lundberg GD, Musacchio RA. Assessing, controlling, and assuring the quality of medical information on the Internet: Caveant lector et viewor--Let the reader and viewer beware. JAMA 1997;277:1244-5.

10. Jones IA, Togashi RC, Thomas Vangsness C Jr. The Economics and Regulation of PRP in the Evolving Field of Orthopedic Biologics. Curr Rev Musculoskelet Med 2018;11:558-65.

11. Alcerro JC, Lavernia CJ. Stem Cells and Platelet-rich Plasma for Knee Osteoarthritis: Prevalence and Cost in South Florida. J Am Acad Orthop Surg 2019;27:779-83.

12. Available at: https://www.transparencymarketresearch. com/platelet-rich-plasma-market.html [Accessed: June 24, 2019].

13. Search Engine Market Share Turkey. Available at: https:// gs.statcounter.com/search-engine-market-share/all/turkey

14. Eysenbach G, Köhler C. How do consumers search for and appraise health information on the world wide web? Qualitative study using focus groups, usability tests, and in-depth interviews. BMJ 2002;324:573-7.

15. Information Triage. Available at: https://www.pewresearch. org/internet/2013/01/15/information-triage/ [Accessed: June 24, 2019].

16. Ghodasra JH, Wang D, Jayakar RG, Jensen AR, Yamaguchi KT, Hegde VV, et al. The Assessment of Quality, Accuracy, and Readability of Online Educational Resources for Platelet-Rich Plasma. Arthroscopy 2018;34:272-8.

17. Le ADK, Enweze L, DeBaun MR, Dragoo JL. Platelet-Rich Plasma. Clin Sports Med 2019;38:17-44.

18. Kelly FB. Platelet-rich plasma (PRP). Published 2011. Available at: http:// orthoinfo.aaos.org/topic.cfm? topic1/4A00648. [Accessed: March 1, 2016].

19. Roberts H, Zhang D, Dyer GS. The Readability of AAOS patient education materials: Evaluating the progress since 2008. J Bone Joint Surg [Am] 2016;98:e70.

20. Schumaier AP, Kakazu R, Minoughan CE, Grawe BM. Readability assessment of American Shoulder and Elbow Surgeons patient brochures with suggestions for improvement. JSES Open Access 2018;2:150-4.

21. Minoughan C, Schumaier A, Kakazu R, Grawe B. Readability of sports injury and prevention patient education materials from the American Academy of Orthopaedic Surgeons Website. J Am Acad Orthop Surg Glob Res Rev 2018;2:e002.

22. Yi MM, Yi PH, Hussein KI, Cross MB, Della Valle CJ. Readability of patient education materials from the web sites of orthopedic implant manufacturers. J Arthroplasty 2017;32:3568-72.

23. Wang SW, Capo JT, Orillaza N. Readability and comprehensibility of patient education material in handrelated web sites. J Hand Surg Am 2009;34:1308-15.

24. Cotugna N, Vickery CE, Carpenter-Haefele KM. Evaluation of literacy level of patient education pages in health-related journals. J Community Health 2005;30:213-9.

25. Demiris G, Afrin LB, Speedie S, Courtney KL, Sondhi M, Vimarlund V, et al. Patient-centered applications: use of information technology to promote disease management and wellness. A white paper by the AMIA knowledge in motion working group. J Am Med Inform Assoc 2008;15:8-13

26. Dy CJ, Taylor SA, Patel RM, McCarthy MM, Roberts TR, Daluiski A. Does the quality, accuracy, and readability of information about lateral epicondylitis on the internet vary with the search term used? Hand (N Y) 2012;7:420-5.

27. Hinds RM, Gottschalk MB, Muppavarapu RC, Naik AA, Yang SS, Capo JT. Assessment of the accuracy of online information regarding trigger finger. J Hand Surg Asian Pac Vol 2016;21:193-8.

28. Sezgin EA, Atik OŞ. Are orthobiologics the next chapter in clinical orthopedics? A literature review. Eklem Hastalik Cerrahisi 2018;29:110-6.

29. Atik OŞ. Every new technique either conservative or surgical is good? Eklem Hastalik Cerrahisi 2019;30:183-4.

30. Kozanhan B, Tutar MS. Readability of patient education texts presented on the internet in the field of anesthesiology. Turkiye Klinikleri J Anest Reanim 2017;15:63-70. 